SHORT REPORT

\title{
Volumetric MRI study of the caudate nucleus in patients with dementia with Lewy bodies, Alzheimer's disease, and vascular dementia
}

\author{
R Barber, I McKeith, C Ballard, J O'Brien
}

J Neurol Neurosurg Psychiatry 2002;72:406-407

Objectives: To determine whether parkinsonian symptoms in dementia with Lewy bodies (DLB) are associated with greater atrophy of the caudate nucleus in comparison with patients with Alzheimer's disease (AD) and vascular dementia (VaD).

Methods: Tl weighted MR scans were acquired in elderly patients with $D L B, A D, V a D$, and healthy controls. Normalised volumetric measurements of the caudate nucleus were obtained and parkinsonian symptoms rated using Hoehn and Yahr staging.

Results: There were no significant differences in the volume of the caudate nucleus between patients with dementia. However, the left caudate volume was significantly reduced in $A D$ and $D L B$ compared with controls. Parkinsonian symptoms did not correlate with caudate nucleus volume.

Conclusions: Parkinsonian symptoms in DLB may be more closely coupled to neurochemical rather than structural changes in the caudate nucleus, and volumetric MRI analysis of caudate nucleus does not discriminate between patients with $D L B, A D$, and $V a D$.

C linically, dementia with Lewy bodies (DLB) is recognised by the triad of fluctuating cognitive impairment, visual hallucinations, and parkinsonian symptoms. ${ }^{1}$ A key pathological feature of DLB is the presence of Lewy bodies in the neocortex, limbic cortex, and subcortical nuclei. ${ }^{1}$ Lewy body pathology in the subcortical areas, particularly the striatum, is associated with a reduction in nigrostriatal dopaminergic neurons and activity. Recently, defects in the integrity of the nigrostriatal dopamine pathway in DLB compared with Alzheimer's disease (AD) have been demonstrated using functional neuroimaging with ligands developed for presynaptic and postsynaptic dopaminergic systems. ${ }^{23}$ These differences are consistent with known neurochemical differences between AD and DLB in the caudate nucleus and putamen. However, it is not known whether these differences are associated with evidence of differential atrophy in the caudate nucleus on structural neuroimaging and whether caudate nucleus atrophy correlates with the severity of parkinsonian symptoms in dementia.

\section{METHODS \\ Subjects}

Brain MRI was acquired in patients with DLB $(n=26 ; 19$ men, mean age 75.8 years, mean duration of illness 36.7 months, mini mental state examination $(\mathrm{MMSE})=14), \mathrm{AD}(\mathrm{n}=21$, eight men, mean age 76.9 years, mean duration 41.3 months, MMSE $=16)$, vascular dementia (VaD) $(n=18,10$ men, mean age 77.5 years, mean duration 39.7 months, MMSE $=18$ ), and healthy controls ( $\mathrm{n}=25,14$ men, mean age 77.5 years, MMSE $=28$ ). Subjects were community dwelling and clinical diagnosis was made blind to MRI using standardised criteria: NINCDS/ADRDA ${ }^{4}$ for $\mathrm{AD}$, consensus criteria ${ }^{1}$ for DLB and NINDS-AIREN ${ }^{5}$ for VaD. Pathological confirmation of diagnosis has since been acquired for six patients. Normal controls were recruited from among the spouses and friends of patients with dementia. After complete description of the study to the subjects and their families, written informed consent was obtained.

\section{Clinical assessments}

Cognitive function was measured using the Cambridge cognitive examination (CAMCOG). ${ }^{6}$ which incorporates the MMSE. ${ }^{7}$

Parkinsonian symptoms were rated using the Hoehn and Yahr staging. ${ }^{8}$

\section{MRI acquisition}

Whole brain Tl weighted three dimensional magnetisation prepared rapid acquisition gradient echo MPRAGE turbo flash sagittal sequence was acquired ( slice thickness $=1 \mathrm{~mm}$ ) using a 1.0 Tesla Siemens Magnetom Impact MRI scanner.

\section{Volume estimation of caudate nucleus}

Images were transferred to a workstation and analyzed using ANALYZE (Version 7.5.5) software. Data was reformatted into coronal slices (slice thickness $=1 \mathrm{~mm}$ ) and aligned perpendicular to the long axis of the hippocampus. All measurements were conducted blind to diagnosis (by RB). Intrarater reliability was assessed by measuring seven subjects on three occasions. The mean coefficient of variation was $2.6 \%$.

Standardised measurements of the area of the left and right caudate nucleus (in $\mathrm{mm}^{2}$ ) was obtained on every fourth slice for a length of $20 \mathrm{~mm}$, starting from the first slice showing the head of the caudate nucleus. Volume estimation (in $\mathrm{mm}^{3}$ ) was calculated by summing the areas outlined and multiplying by 4 .

Measurements were normalised to the midsagittal intracranial area to control for variation in head size. Final volumes were expressed as a normalised ratio (units $\mathrm{mm}^{3} / \mathrm{mm}^{2}$ ).

\section{Statistical analysis}

Continuous variables were assessed using analysis of variance (ANOVA) with post hoc Bonferroni tests. Kruskal-Wallis ANOVA test was used for non-parametric data. All volumetric comparisons were made using the normalised ratio. Correlations were examined using Spearman's rank order correlation coefficient $(r)$. All statistical tests were regarded as significant at $\mathrm{p}<0.05$.

Abbreviations: DLB, dementia with Lewy bodies; AD, Alzheimer's disease; $\mathrm{VaD}$, vascular dementia 
Table 1 Summary of normalised $\mathrm{CN}$ volumes by diagnostic group

\begin{tabular}{lll}
\hline & Right CN (SD) & Left CN (SD) \\
\hline $\mathrm{DLB}$ & $11.7(2.0)$ & $11.4(1.7)^{*}$ \\
$\mathrm{AD}$ & $11.8(2.4)$ & $11.1(2.6)^{*}$ \\
$\mathrm{VaD}$ & $11.7(1.7)$ & $11.8(1.7)$ \\
Controls & $13.0(2.1)$ & $13.2(1.8)$ \\
\hline
\end{tabular}

${ }^{*} \mathrm{p}<0.05 ;{ }^{*}{ }^{*} \mathrm{p}<0.01$

Values expressed as normalised volumes (volume of structure in $\mathrm{mm}^{3}$ /intracranial area in $\mathrm{mm}^{2}$ ) (SD).

$C N$, caudate nucleus; DLB, dementia with Lewy bodies; $A D$

Alzheimer's disease; $\mathrm{VaD}$, vascular dementia.

\section{RESULTS}

Subjects with dementia were of comparable age, duration of illness, $\operatorname{sex}\left(\chi^{2}=5.7, \mathrm{df}=3, \mathrm{p}=0.124\right)$ and cognitive impairment as measured by CAMCOG scores $(\mathrm{DLB}=48, \mathrm{AD}=57$, $\mathrm{VaD}=60 ; \mathrm{p}=0.110)$ or MMSE $(\mathrm{p}=0.66)$. As expected, patients with DLB had higher Hoehn and Yahr scores compared with all other groups (median scores: $\mathrm{DLB}=3, \mathrm{AD}=0, \mathrm{VaD}=0$; $\left.\chi^{2}=75, \mathrm{df}=3, \mathrm{p}<0.001\right)$.

\section{Volumetric comparison of caudate nucleus in different groups}

There were no significant differences in the normalised volume of the caudate nucleus between patients with dementia $(\mathrm{DLB}=11.6, \mathrm{AD}=11.5, \mathrm{VaD}=11.8 ; \mathrm{p}=0.887)$. However, as summarised in table 1 , both the left and right caudate nucleus were smaller in patients with dementia compared with normal age matched controls, although this differences was only significant for the left caudate nucleus in patients with DLB $(11.4 v 13.2, \mathrm{p}=0.01)$ and $\mathrm{AD}(11.1 v 13.2, \mathrm{p}<0.01)$.

\section{Correlation between caudate nucleus volumes and parkinsonian symptoms}

In all patients with dementia there was no correlation between caudate nucleus volume and parkinsonian symptoms (DLB $r=0.183, \mathrm{p}=0.380 ; \mathrm{AD} \quad r=-0.074, \mathrm{p}=0.750 ; \mathrm{VaD}$ $r=-0.023, \mathrm{p}=0.927)$.

\section{DISCUSSION}

There were no detectable structural differences on MRI in the volume of the caudate nucleus between patients with DLB, $\mathrm{AD}$, and $\mathrm{VaD}$, and the volume of the caudate nucleus did not correlate with parkinsonian symptoms.

By contrast with the differences reported using functional imaging, ${ }^{2}{ }^{3}$ volumetric analysis of the caudate nucleus on MRI did not discriminate between diagnostic groups. As recently shown, ${ }^{9}$ compared with controls there was evidence of atrophy in $\mathrm{AD}$ although the similar finding in patients with DLB suggests that this not a specific feature, inferring this measure has little clinical utility in the discrimination of patients with DLB, AD, or VaD.

Overall, this study indicates that parkinsonian symptoms in DLB are unlikely to be specifically associated with atrophy of the caudate nucleus, and that caudate atrophy on MRI itself does not explain differences in parkinsonian symptoms between $\mathrm{DLB}, \mathrm{AD}$, and VaD. The findings suggest that parkinsonian symptoms in DLB are possibly more closely coupled to neurochemical rather than structural changes in the caudate nucleus. Therefore, functional neuroimaging techniques may prove to be a more sensitive method of examining differences in striatal pathology than MRI.

Limitations of this study include the use of antemortem diagnoses, that volume estimation was restricted to the anterior portion of the caudate nucleus, and the clinical measure of parkinsonian symptoms may have been too insensitive to correlate with MRI indices. Strengths included the recruitment of a community population of subjects of comparable age, duration of illness, and level of cognitive impairment, and the use of standardised diagnostic criteria and normalised volumetric measurements.

These preliminary findings need replication in a larger cohort, ideally combining both MRI and functional neuroimaging techniques with postmortem follow up.

\section{ACKNOWLEDGEMENTS}

We thank Professor R Perry and Dr E Jaros for neuropathological diagnoses and Dr A Gholkar and Mr Philip English for performing MRI. The research was supported by a grant from the Northern and Yorkshire Regional Health Authority and Medical Research Council, UK.

\section{Authors' affiliations}

R Barber, I McKeith, C Ballard, J O'Brien Institute for the Health of the Elderly, Newcastle General Hospital, Newcastle upon Tyne, NE4 6BE, UK

Correspondence to: $\operatorname{Dr} R$ Barber, Akenside Unit, Centre for the Health of the Elderly, Newcastle General Hospital, Newcastle upon Tyne, NE4 6BE, UK; Robert.Barber@ncl.ac.uk

Received 27 April 2001

In revised form 11 October 2001

Accepted 5 November 2001

\section{REFERENCES}

1 McKeith IG, Galasko D, Kosaka K, et al. Consensus guidelines for the clinical and pathological diagnosis of dementia with Lewy bodies (DLB): report of the Consortium on Dementia with Lewy Bodies International Workshop. Neurology 1996;47:1113-24.

2 Donnemiller E, Heilmann J, Wenning G, et al. Brain perfusion scintigraphy with $99 \mathrm{mTc}-\mathrm{HMPAO}$ or $99 \mathrm{mTc}-\mathrm{ECD}$ and 1231 -beta-CIT single-photon emission tomography in dementia of the Alzheimer-type and diffuse Lewy body disease. Eur J Nucl Med 1997;24:320-5.

3 Walker Z, Costa D, Ince $P$, et al. In-vivo demonstration of dopaminergic degeneration in dementia with Lewy bodies. Lancet 1999;354:646-7.

4 McKhann G, Drachman D, Folstein M, et al. Clinical diagnosis of Alzheimer's disease: report of the NINCDS-ADRDA Work Group under the auspices of Department of Health and Human Service Task Force on Alzheimer's disease. Neurology 1984;34:939-44.

5 Roman GC, Tatemichi T, Erkinjuntti T, et al. Vascular dementia: diagnostic criteria for research studies. Report of the NINDS-AIRENS international workshop. Neurology 1993;43:250-60.

6 Roth M, Tym E, Mountjoy C, et al. CAMDEX. A standardised instrument for the diagnosis of mental disorder in the elderly with special reference to the early detection of dementia. Br J Psychiatry 1986;149:698-709.

7 Folstein M, Folstein S, McHugh P. "Mini-mental state": a practical method for grading the cognitive state of patients for the clinician. $J$ Psychiatr Res 1975;12:189-98.

8 Hoehn MM, Yahr MD. Parkinsonism: onset, progression, and mortality. Neurology 1967; 17:427-42.

9 Rombouts SA, Barkhof F, Witter MP, et al. Unbiased whole-brain analysis of gray matter loss in Alzheimer's disease. Neurosci Lett 2000;285:231-3. 